\title{
CENTRAL SEROUS CHORIORETINOPATHY IN PREGNANCY
}

\author{
Gunasena G.G.A ${ }^{1}$, Kumarasiri J.M², Tillekeratne L.P.A ${ }^{3}$
}

\section{INTRODUCTION}

Numerous physiologic effects occur within the body during pregnancy, and the eye is no exception. Most of the ocular changes are specifically associated with the pregnancy itself, and they rarely disturb the normal visual function. These ocular changes are more commonly transient but occasionally, permanent. The ocular effects of pregnancy may be divided into physiologic changes, pathologic conditions and modifications of preexisting conditions like diabetes mellitus. Pregnancy is associated with changes in corneal sensitivity and thickness, decreased intraocular pressure, and visual field defects. Pathological conditions known to develop during pregnancy include pregnancy-induced hypertension (pre-eclampsia), vascular disorders, idiopathic intracranial hypertension, and central serous chorioretinopathy (CSCR) (1).

Although not typical, central serous chorioretinopathy has been reported to occur during pregnancy. It is an idiopathic disorder characterized by a local serous detachment of the neurosensory retina at the macula, secondary to leakage from the choriocapillaries through one or more hyper-permeable RPE (retinal pigment epithelium) sites.

We report a case of 36 year old mother in her second pregnancy developing bilateral CSCR at the beginning of third trimester.

\footnotetext{
1. District Base Hospital, Rikillagskada, Sri Lanka

2. Castle Street Hospital for Women, Colombo 08, Sri Lanka

1. Teaching Hospital, Kandy, Sri Lanka
}

Correspondence: Dr. Gunasena G.G.A

E-mail: asankagunasena@gmail.com

Competing interests: None

\section{CASE REPORT}

A 36-year-old mother in her second pregnancy presented with visual symptoms at twenty-eight weeks of gestation. The condition first appeared on the right eye with blurred and distorted vision (metamorphopsia), flashes of light (photopsia), and reduced visual acuity. The condition gradually progressed to involve both eyes and visual acuity drastically declined over time to near blindness. She was seen by the ophthalmology unit Colombo and the diagnosis of central serous chorioretinopathy was made by clinical evaluation of the macula with fundoscopy. Because of the dramatically worsening ocular changes within a short period of time, a decision was made to deliver the baby at 32 weeks of gestation by caesarean section after administration of dexamethazone for fetal lung maturity. After the delivery, she was managed conservatively at ophthalmology unit Colombo. She made an excellent spontaneous recovery postpartum within two months.

\section{DISCUSSION}

Central serous chorioretinopathy (CSCR) is a localized retinal detachment of macular area. It is caused by a localized defect of the underlying retinal pigment epithelium (RPE) resulting in fluid of choroidal origin coming to subretinal space causing serous macular detachment.

This condition affects young individuals between the ages of 20-50 years. The disease is seen predominantly in males as compared to females; the ratio is reported to be 10:1(2). Women with CSCR tend to be older, except in pregnancy. It is unilateral in $90 \%$ of patients. Increased incidence of CSCR has been observed in conditions like type A personality, pregnancy, and Cushing's syndrome.
In these conditions, the increase in the level of endogenous cortisol is thought to be involved in the pathogenesis (3). Other risk factors implicated in the pathogenesis include stress, steroids, hypertension, and SLE.

The presenting symptoms are unilateral blurring of vision (rarely bilateral), metamorphopsia, micropsia, mild dyschromatopsia, a relative positive scotoma, photopsia, occasional macropsia, and moderately reduced visual acuity (2). Although more common in the third trimester, it may also occur during the first or second trimesters. Most CSCR heals spontaneously in 4-8 weeks postpartum, with recovery of visual acuity within 3 to 6 months. But RPE alterations, metamorphopsia, and central visual field changes may rarely persist with severe visual loss reported in 5\% of CSCR. Recurrences are known to occur in future pregnancies and $10 \%$ have three or more recurrences. Half of the recurrences occur within one year but may occur up to 10 years after the first episode (2).

The clinical findings can be demonstrated on the Amsler grid. Ophthalmoscopy reveals a circumscribed round or oval area of retinal elevation in the macula area. Slitlamp biomicroscopy typically shows serous elevation of the macula. The diagnosis of CSCR is confirmed by OCT (optical coherence tomography) and fluorescein angiography, which demonstrates the site of RPE detachment and the site of leakage of serous fluid from the RPE into the subretinal space. Although there is no evidence that fluorescein angiography during pregnancy adversely affects the fetus, there seems to be no indication to perform fluorescein angiography during pregnancy in cases of uncomplicated CSCR in view of the universal spontaneous resolution and good visual acuity outcome of the cases reported in the literature (4). 
The underlying mechanism of CSCR during pregnancy remains unclear. It is believed that the raised levels of endogenous steroid Cortisol could set off a chain of events that alter the blood-retinal barrier, choriocapillaries, and retinal pigment epithelium, resulting in focal areas of increased permeability, and consequently precipitating CSCR (5).

The treatment of CSCR is controversial. No medication has been shown to be effective in the management; in fact, the use of systemic steroid has resulted in the development of CSCR and worsening of existing CSCR (6). Micropulse Diode Laser to the RPE site of leakage underlying the CSCR shortened the duration of CSCR by 2 months and also reduced the recurrence rate (7). The possible mechanism of laser treatment is debridement of diseased retinal pigment epithelium permitting ingrowths of surrounding healthy RPE and resolution of CSCR. Since most CSCR resolve spontaneously, immediate laser treatment is not advisable. Patients are also advised to manage their stress adequately and get at least 7-8hrs of sleep per day.

\section{REFERENCES}

1. Pilas-Pomykalska M, Czajkowski J, Oszukowski P. Ocular changes during pregnancy. Ginekol Pol. 2005 Aug;76(8):655-60.

2. D Hussain, JD Gass. Idiopathic central serous chorioretinopathy. Current ophthalmology. 1998; 46(3): 131-137.

3. Bouzas EA, Scott MH, Mastorakos G,
Chrousos GP, Kaiser-Kupfer MI. Central serous chorioretinopathy in endogenous hypercortisolism. Arch Ophthalmol 1993; 111:1229-32.

4. Sunness JS. Pregnancy and the eye. Ophthalmol Clin North Am. 1992; 5:623640.

5. Al-Mujaini A, Wali U, Ganesh A, Montana C. Natural course of central serous chorioretinopathy without subretinal exudates in normal pregnancy. Can $\mathrm{J}$ Ophthalmol. Vol. 43, No. 5, 2008

6. Gass JDM, Little HL. Bilateral bullous exudative retinal detachment complicating idiopathic central serous chorioretinopathy during systemic corticosteroid therapy. Ophthalmology 1995;102:737-47

7. Spitznas ML. Central serous retinopathy. In: Ryan SJ, editor. Retina. 2nd ed. St. Louis: CV Mosby; 1994. p 1167. 